\title{
Study on the flavonoids and properties of extraction and purification process
}

\author{
Ma Xiaogen, Zhou Huiyan \\ Jiyuan Vocational and Technical College, Jiyuan454650, China
}

Keywords: extraction and purification; extraction solvent; process optimization; extraction rate; flavonoids

\begin{abstract}
Flavonoids have very important medical value. They have many functions, such as anti-inflammatory and anti-tumor virus. They mainly come from the photosynthesis of plants, so they can extract flavonoids from plants, such as clover. The material structure and physical properties of flavonoids were analyzed. The extraction methods of flavonoids were discussed. Finally, the extraction process of total flavonoids from Trifolium repens was studied by using ethanol as solvent. The optimum extraction technology of single factor experiments and orthogonal experiment optimization of total flavonoids from Radix Tetrastigmae was 60\% ethanol concentration, the extraction time is 1.5 hours, and the suitable process conditions in this optimization, which can get higher flavonoids extraction and purification rate, thereby improving the production efficiency of flavonoids.
\end{abstract}

\section{Introduction}

Clover is a popular Chinese herbal medicine, which has the functions of clearing away heat and toxic materials, expelling wind, resolving phlegm, promoting blood circulation and relieving pain function. Pharmacological studies have shown that the flavonoids have antitumor, hepatoprotective and pharmacological effects. But now few studies have been reported on the extraction and purification process of Flavonoids from green clover. For better development and utilization of the fund.

Therefore, it is of great significance to study the extraction and purification process of flavonoids. Ethanol is a kind of important organic solvent extraction, its low toxicity, easy recovery, high absorption efficiency, is widely used in the purification of various industries and fields, the use of ethanol as organic solvent of flavonoids, rate of the effects of ethanol concentration and extraction time on the extraction of flavonoids, flavonoids and it can provide technical support for production and processing.

\section{Analysis of the structure and physical properties of flavonoids}

General flavone in compounds of the form, which belongs to the biological class of natural compounds, the main source of plant photosynthesis, so the plant is widely distributed, the higher content of Leguminosae, Composited and aromatic plants, etc.. Flavonoids mainly refer to a class of compounds whose basic parent is 2-, and its structure is shown in Figure 1. 


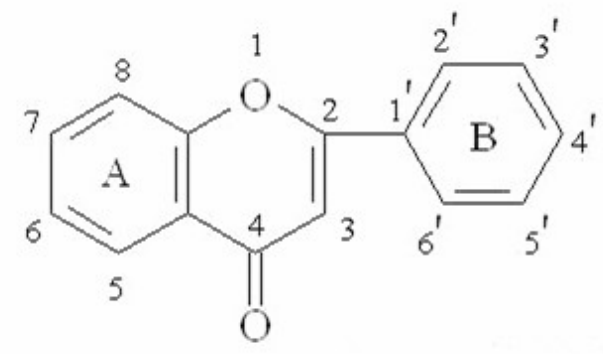

Fig. 1 The structure of 2- phenyl ketone

As shown in Figure 1, the flavonoids is generally a multi crystal structure, its color and structure, color molecules cross conjugated system and auxochromes, under visible light, flavonoids can appear pale yellow - yellow, yellow - orange, yellowish, medicinal effect of flavonoids is very good there are two main aspects of the role.

(1) Anti-inflammatory effect. Studies have shown that flavonoids can reduce the permeability of capillary effect, so as to achieve anti-inflammatory properties, and some studies show that flavonoids can inhibit the synthesis of an enzyme, so as to achieve anti-inflammatory effects, this enzyme is ester oxidase, is the biosynthesis of prostate. The experiments in mice showed that flavonoids could inhibit the swelling and inflammation of mice caused by xylene, and could inhibit the release of glucose release enzyme.

(2) Antitumor effect. Flavonoid has anti-tumor effect, the study showed that flavonoids can inhibit tumor cell proliferation, or kill tumor cells to achieve anti-tumor effect, which can also be enhanced by other substances in activity indirectly inhibit the growth and reproduction of tumor cells, or even eliminate some carcinogenic toxic chemical carcinogens.

\section{The extraction method and technology of flavonoids}

The extraction methods of physical and chemical methods, chemical methods mainly include extraction, alcohol dilute alkaline organic solvent extraction method, physical method mainly includes water boiling method, ultrasonic extraction method, is the most widely used organic solvent method, organic solvent is used in methanol, ethanol, ether and acetone etc.. Methanol and other organic solvents are more toxic, but ethanol is less toxic, simple equipment, easy to recycle, and the penetration of plant cells is strong, and the extraction effect is good. Therefore, this study mainly uses ethanol to extract flavonoids, in which the materials and specifications used are as shown in Table 1.

Table 1 The equipment and specifications used in the experiment

\begin{tabular}{|c|c|}
\hline Experimental material & Specifications \\
\hline rutin & Greater than $95 \%$ \\
\hline Absolute ethanol & Analytical grade purity \\
\hline Sodium nitrite & Analytical grade purity \\
\hline $\mathrm{AL}(\mathrm{NO})_{3}$ & Analytical grade purity \\
\hline $\mathrm{NaOH}$ & Analytical grade purity \\
\hline Trifoliate green & Analytical grade purity \\
\hline
\end{tabular}

As shown in Table 1, the clover roots were crushed, take a certain amount of powder, placed in the flask, it is easy to type of alcohol in flask, and at a certain temperature, placed in a water bath, so that it can keep a constant temperature, the reflux condensation and filtration. During the extraction time, the filter residue was extracted and the extract was combined. The extracted ethanol was easily packed in a $100 \mathrm{~mL}$ container for determination and analysis. 


\section{Optimization Analysis of extraction and purification process of flavonoids}

In order to optimize the extraction and purification of flavonoids, flavonoids according to different process conditions to optimize the extraction rate, the reasonable technological conditions, the extraction rate of Flavonoids from Radix Tetrastigmae as an example, in which the extraction rate of flavonoids can be calculated according to the following formula

$$
P=\frac{C \times 10 \times 100}{0.5 \times W \times 1000}
$$

Which means that the total flavonoids concentration in the sample, 10 and 100 mean dilution times, and 0.5 means that the amount of solution to be measured is $0.5 \mathrm{~mL}$, indicating the quality of trefoil, indicating the magnitude of the unit conversion. Through the experiment, the extraction effect of different ethanol concentration was tested, and the test results were shown as shown in Figure 2

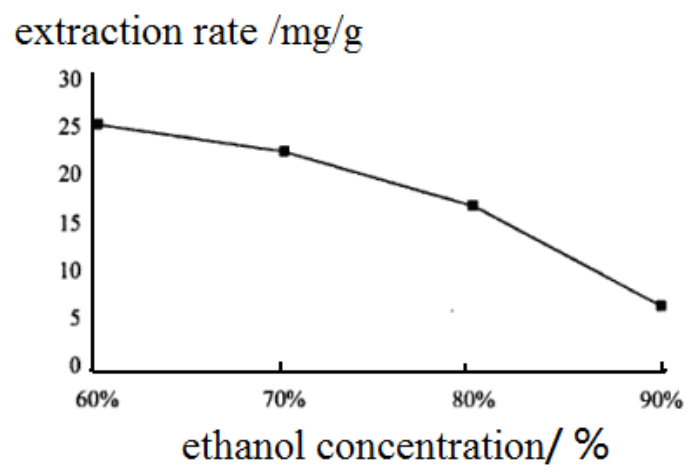

Fig. 2 The relationship between ethanol concentration and extraction rate

As shown in Figure 2, the extraction rate of flavonoids varied with the concentration of ethanol, and the curve results show that with the increase of the ethanol concentration, the extraction rate of flavonoids decreased gradually when the concentration of ethanol was $60 \%$, the highest rate of acquisition of flavonoids, so in the process of optimization, needs to consider the concentration of ethanol.

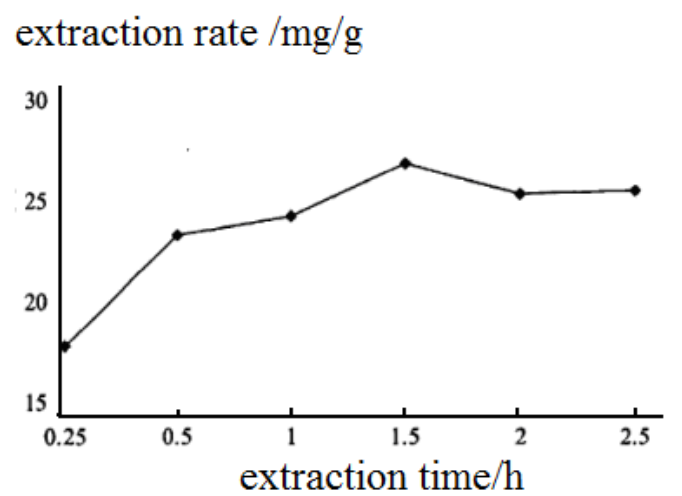

Fig. 3 relation curve between extraction time and extraction rate

As shown in Figure 3, it represents the variation curve of the extraction rate of flavonoids with the extraction time, the curve results show that with the increase of extraction time, increase the extraction rate of flavonoids, extraction rate reached the highest peak at $1.5 \mathrm{~h}$, after 1.5 hours, both inside and outside the system to reach equilibrium concentration, not conducive to the extraction and filtration therefore, the extraction efficiency decreased in flavonoids extraction and purification process, must be considered in order to achieve the best extraction time, the extraction and purification effect.

\section{Conclusion}

In order to improve the efficiency of extraction and purification of flavonoids, based on the analysis of the properties of flavonoids, extraction of flavonoids were optimized. The optimization 
method mainly adopts single factor and orthogonal experiment method, through the investigation of flavonoids extraction rate, the feasibility of the process. Due to the time limit, the effect of ethanol concentration and extraction time on the extraction rate was mainly tested. The experimental results showed that the extraction rate of flavonoids was the best when the concentration of ethanol was $60 \%$ and the extraction time was 1.5 hours. Therefore, in the extraction of flavonoids, you need to choose the appropriate process conditions, in order to obtain higher extraction and purification rate of flavonoids, improve the productivity of flavonoids.

\section{References}

[1] Yao Xingcun, Shu Liuquan, Ding Qunwen. The antioxidation and stability of the extracts from Porphyra yezoensis [J]. Science and technology of food industry,2013(06).

[2] Wang Chengwen, Ji Minghui, Chen Guangying, etc.. The antioxidant activity and stability of the tropical root extract mokara [J]. Science and technology of food industry,2013 (05).

[3] Zhao Furong, KangJian, ChenYinru. The ultrasonic and microwave assisted extraction of Flavonoids from seabuckthorn leaves[J]. Anhui Agricultural Sciences, 2012 (30).

[4] Sun Tianyu, Yang Hongzhi. Study on the optimum process of Extracting Total Flavonoids from seabuckthorn leaves by ultrasonic wave [J]. Journal of Heilongjiang Bayi Agricultural University,2012 (03).

[5] Jia Na, Kong Baohua, Zhang Hongtao. Study on the extraction and antioxidant activity of blackcurrant anthocyanins [J].Food science,2011 (16).

[6] Wang Ruixue, Sun Yang, Qian Fang. The antioxidant peptide and its research progress [J]. Food science and technology, 2011 (05).

[7] Lu Xinsheng, Zhang Hailing, Liu Boqu, Wang Yaling. The chemical composition and medicinal value of Tibetan snow lotus Ru Hu Gou [J]. northern horticulture, 2011 (06).

[8]Wan Lixiu Xiao, Xu Yujuan, Jiang Aiminetc.. The citrus flavonoids before and after purification antioxidant comparative study [J]. Food science,2011 (05).

[9] SunLi, Luo Qiang, ZhangLi,etc.. The effects of Trollius flavonoids on the growth and apoptosis of A549 cell [J]. Journal of China Gerontology,2011(01).

[10] Ma Hongfeng, Gao Limei, Zhao Guangyun,etc.. The colorimetric determination of total flavonoids in chestnut flowers[J]. Chinese Modern Chinese medicine, 2010 (10). 